\title{
Fibrin glue as a protective biomembrane for a duodenal ulcer vessel
}

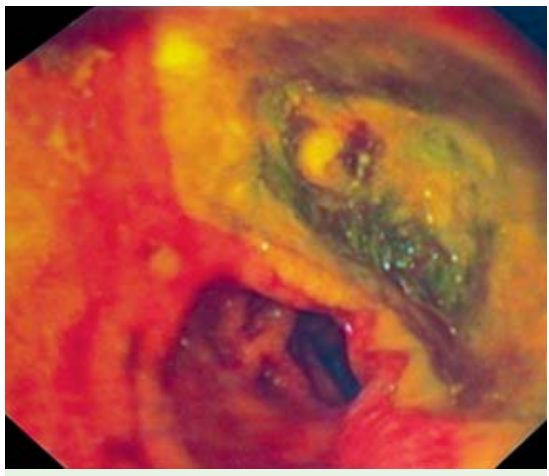

Fig. 1 Large ulceration in the posterior duodenal bulb wall with the crater base mainly consisting of muscle fibers. A 2-3-mm, nonbleeding but pulsing vessel is seen within the ulcer crater.

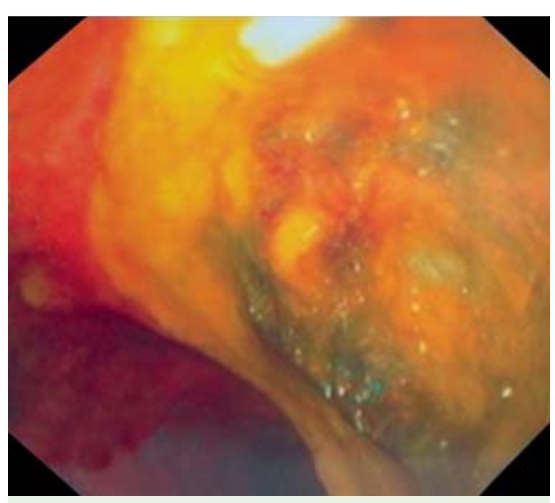

Fig. 2 The ulcer after initial fibrin glue application.

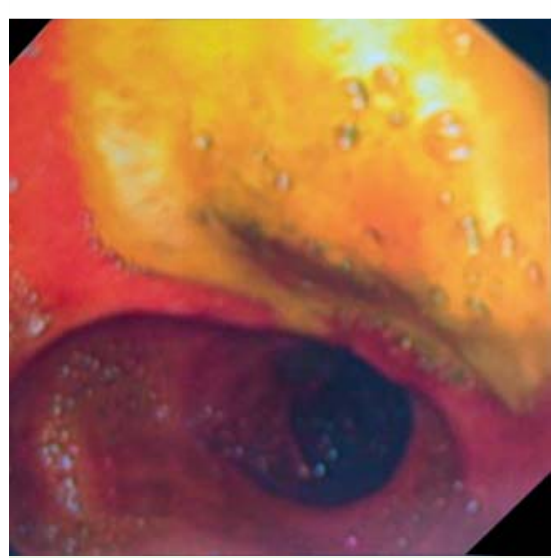

Fig. 3 The ulcer after the second fibrin glue session.

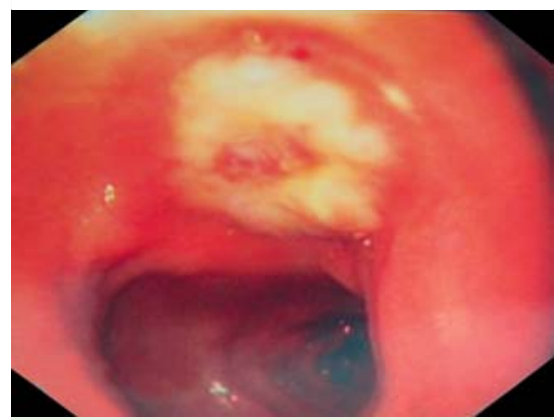

Fig. 4 Gastroscopy (1 month after the bleeding) showing healing of the ulcer.

Bleeding peptic ulcers are a common emergency, and contemporary endoscopic treatments include injection, thermal, and mechanical therapy [1]. We describe the use of fibrin glue as a protective biomembrane over a large, recently bleeding visible duodenal ulcer vessel and crater, at risk of perforation.

A 44-year-old woman was admitted for hematemesis and hypovolemic shock. She had been receiving $100 \mathrm{mg}$ acetylsalicylic acid daily, which was the likely causative agent. She had homozygous $\beta$-thalassaemia, hemosiderosis, hepatomegaly, viral hepatitis $\mathrm{C}$, type 2 diabetes mellitus, chronic renal failure under peritoneal dialysis, cardiac insufficiency due to restrictive cardiomyopathy treated with pacemaker, and hypothyroidism. She had undergone splenectomy and had her gallbladder removed.

The patient was resuscitated and emergency gastroscopy carried, out but it was impossible to identify bleeding vessels due to the excessive amount of bleeding and clots. After 12 hours a repeat gastroscopy identified a 2-cm FIIa-G ulcer on the posterior wall of the duodenal bulb; the crater base was formed almost entirely of muscle fibers ( $\bullet$ Fig. 1).

A large nonbleeding, but pulsing, vessel (diameter 2-3 mm) was observed in the crater of the ulcer. Any conventional measure to occlude the vessel was judged impossible, while an emergency operation was contraindicated. Fibrin glue as a bio-barrier was considered and $2 \mathrm{~mL}$ of the glue was applied on the crater through a double lumen catheter, in order to protect the exposed vessel from erosion (๑ Fig. 2).
At 3 days, another gastroscopy revealed an intact, protective biofilm and an ongoing healing process. A further layer of fibrin glue was applied ( $\bullet$ Fig. 3 ).

After 1 month, a final gastroscopy was done to evaluate the stage of healing of the ulcer, which was noted to be adequate ( $\bullet$ Fig. 4), and the patient was thus discharged.

Fibrin glue is used in fistulas, either alone or with endoclips [2,3], because in addition to its mechanical role (occlusion), it has a role in wound healing [4]. The idea of using fibrin glue arose due to the very poor general condition of the patient and the fact that traditional endoscopic methods could lead to ulcer perforation. Fibrin glue seemed the ideal material to form a biomembrane (easily applied, impermeable to gastroduodenal fluids, enhanced wound healing).

The application of fibrin glue as a protective biomembrane over an exposed vessel in a duodenal ulcer was deemed successful in the present case. Of course, this technique has only been used in one patient and its overall effectiveness remains to be explored.

\section{Competing interests: None}

Endoscopy_UCTN_Code_TTT_1AO_2AN

G. Germanidis ${ }^{1}$, T. S. Papavramidis ${ }^{2}$, K. Mantzoukis ${ }^{1}$, K. Sapalidis ${ }^{2}$, D. Divanis $^{1}$, I. Kalevrosoglou ${ }^{1}$, E. Chassapopoulou $^{1}$, P. Nikolaidis ${ }^{1}$

1 1st Department of Internal Medicine, AHEPA University Hospital, Aristotle University of Thessaloniki, Thessaloniki, Greece

2 3rd Department of Surgery, AHEPA University Hospital, Aristotle University of Thessaloniki, Thessaloniki, Greece

\section{References}

1 Gralnek IM, Barkun AN, Bardou M. Management of acute bleeding from a peptic ulcer. N Engl J Med 2008; 359: 928 - 937

2 Papavramidis TS, Kotzampassi K, Kotidis E et al. Endoscopic fibrin sealing of gastrocutaneous fistulas after sleeve gastrectomy and biliopancreatic diversion with duodenal switch. J Gastroenterol Hepatol 2008; 23: $1802-1805$ 
3 Papavramidis ST, Eleftheriadis EE, Papavramidis TS et al. Endoscopic management of gastrocutaneous fistulas after bariatric surgery by using a fibrin sealant. Gastrointest Endosc 2004; 59: 296- 300

4 Rolandelli R, Roslyn JJ. Surgical management and treatment of sepsis associated with gastrointestinal fistulas. Surg Clin North Am 1996; 76: $1111-1122$
Bibliography

DOI $10.1055 / \mathrm{s}-0030-1255982$

Endoscopy 2010; 42: E348 - E349

(c) Georg Thieme Verlag KG Stuttgart · New York . ISSN 0013-726X
Corresponding author

\section{T. Papavramidis}

3rd Department of Surgery, AHEPA University Hospital, Aristotle University of Thessaloniki, Aigaiou 6

54655 Thessaloniki

Greece

papavramidis@hotmail.com 